\title{
Mining data from milk infrared spectroscopy to improve feed intake predictions in lactating dairy cows
}

\author{
J. R. R. Dórea, ${ }^{*}$ G. J. M. Rosa,† K. A. Weld, ${ }^{*}$ and L. E. Armentano*1 \\ *Department of Dairy Science, and \\ †Department of Animal Sciences, University of Wisconsin, Madison 53706
}

\begin{abstract}
Feed intake is one of the most important components of feed efficiency in dairy systems. However, it is a difficult trait to measure in commercial operations for individual cows. Milk spectrum from mid-infrared spectroscopy has been previously used to predict milk traits, and could be an alternative to predict dry matter intake (DMI). The objectives of this study were (1) to evaluate if milk spectra can improve DMI predictions based only on cow variables; (2) to compare artificial neural network (ANN) and partial least squares (PLS) predictions; and (3) to evaluate if wavelength (WL) selection through Bayesian network (BN) improves prediction quality. Milk samples $(\mathrm{n}=1,279)$ from 308 midlactation dairy cows $[127 \pm 27 \mathrm{~d}$ in milk (DIM)] were collected between 2014 and 2016. For each milk spectra time point, DMI $(\mathrm{kg} / \mathrm{d})$, body weight $(\mathrm{BW}, \mathrm{kg})$, milk yield (MY, $\mathrm{kg} / \mathrm{d})$, fat (\%), protein (\%), lactose (\%), and actual DIM were recorded. The DMI was predicted with ANN and PLS using different combinations of explanatory variables. Such combinations, called covariate sets, were as follows: set 1 (MY, $\mathrm{BW}^{0.75}$, DIM, and $\left.361 \mathrm{WL}\right)$; set $2\left[\mathrm{MY}, \mathrm{BW}^{0.75}\right.$, DIM, and $33 \mathrm{WL}$ (WL selected by $\mathrm{BN})]$; set $3\left(\mathrm{MY}, \mathrm{BW}^{0.75}\right.$, DIM, and fat, protein, and lactose concentrations); set $4\left(\mathrm{MY}, \mathrm{BW}^{0.75}, \mathrm{DIM}, 33\right.$ $\mathrm{WL}$, fat, protein, and lactose); set 5 (MY, $\mathrm{BW}^{0.75}$, DIM, $33 \mathrm{WL}$, and visit duration in the feed bunk); set 6 (MY, DIM, and $33 \mathrm{WL}$ ); set 7 (MY, BW ${ }^{0.75}$, and DIM); set-WL (included $361 \mathrm{WL}$ ); and set-BN (included just 33 selected WL). All models (i.e., each combination of covariate set and fitting approach, ANN or PLS) were validated with an external data set. The use of ANN improved the performance of models 2, 5, 6, and $\mathrm{BN}$. The use of BN combined with ANN yielded the highest accuracy and precision. The addition of individual WL compared with milk components (set 2 vs. set 3 ) did not improve prediction quality when using PLS.
\end{abstract}

Received October 16, 2017.

Accepted March 3, 2018.

${ }^{1}$ Corresponding author: learment@wisc.edu
However, when ANN was employed, the model prediction with the inclusion of $33 \mathrm{WL}$ was improved over the model containing only milk components (set 2 vs. set 3 ; concordance correlation coefficient $=0.80$ vs. 0.72 ; coefficient of determination $=0.67$ vs. 0.53 ; root mean square error of prediction 2.36 vs. $2.81 \mathrm{~kg} / \mathrm{d}$ ). The use of ANN and the inclusion of a behavior parameter, set 5 , resulted in the best predictions compared with all other models (coefficient of determination $=0.70$, concordance correlation coefficient $=0.83$, root mean square error of prediction $=2.15 \mathrm{~kg} / \mathrm{d}$ ). The addition of milk spectra information to models containing cow variables improved the accuracy and precision of DMI predictions in lactating dairy cows when ANN was used. The use of BN to select more informative WL improved the model prediction when combined with cow variables, with further improvement when combined with ANN.

Key words: dry matter intake, milk spectra, machine learning

\section{INTRODUCTION}

Feed intake is one of the most important components of feed efficiency in dairy systems. It is, however, a difficult trait to measure in commercial conditions for individual cows. Simple regression equations as proposed by the Agricultural Research Council (ARC, 1980), the Cornell Net Carbohydrate and Protein System (Fox et al., 2004), and the NRC (2001) are widely used as predictors of DMI on commercial farms. Body weight, milk yield, and DIM are commonly used as explanatory variables in these models. However, when a cow's gross feed efficiency increases, such as increases in milk yield due to body fat mobilization without changes in DMI, the use of models only considering milk yield, BW, and DIM could result in poor DMI predictions.

The use of milk spectrum from Fourier transform mid-infrared (FT-IR) spectroscopy has been used to estimate milk components such as milk fatty acids (Soyeurt et al., 2011), milk protein fractions (Soyeurt et al., 2012), milk trait breeding values (Dagnachew et al., 
2013), and methane emissions (Vanlierde et al., 2016). Recently, Dórea et al. (2017) reported that milk fatty acids such as C18:1 are related to plasma nonesterified fatty acid concentration, which is an indicator of body fat mobilization. An accurate prediction of this specific milk fatty acid using FT-IR analysis is already feasible (Soyeurt et al., 2006, Coppa et al., 2010), and it is an example of how milk spectra can provide information about cow metabolism.

Predicting DMI using milk FT-IR was first done by Shetty et al. (2017). These authors compared models to predict DMI using milk yield, BW, and milk components (fat, protein, and lactose) with a model where milk components were replaced by milk spectra. The results did not show a benefit of using the milk spectra. However, the authors used a partial least squares (PLS) approach, which may not be optimal given the high dimension of the predictor variables $(\mathrm{n}=1,060$ wavelengths, WL) and the fact that the relationship between some WL and DMI may be nonlinear. In this scenario, the benefit of including full spectra compared with milk components is not fully explored and might result in a lack of improvement in the prediction quality (Shetty et al., 2017). In addition, Shetty et al. (2017) found no improvement of DMI prediction when the WL included in the model were selected through a method called recursive weighted partial least squares proposed by Rinnan et al. (2014). Other methods have been used for variable selection as reported by Felipe et al. (2015) who used the concept of Markov blanket (MB) to select a smaller set of explanatory variables related to the response variable. The MB learning algorithm is a supervised algorithm implemented in the context of Bayesian networks (BN) and directed acyclic graphs (DAG). The MB of a node is the set of nodes including its parent(s), child(ren), and spouse(s), and is also known as the minimal set of nodes, which makes it conditionally independent in the joint distribution from all the others (Felipe et al., 2015).

Although PLS has been the most used approach for estimation of model parameters in milk FT-IR studies (Soyeurt et al., 2006; Coppa et al., 2010; Eskildsen et al., 2014), the use of machine learning techniques could improve the quality of the predictions due to their ability to model complex relationships between variables, such as nonlinearities and interactions (Gianola et al., 2011). The use of artificial neural networks (ANN), for example, could be an alternative approach to develop predictive models using milk FT-IR. Artificial neural networks were developed based on the human nervous system and consist of interconnected neurons (linear or nonlinear) organized into layers (Bishop, 2006). This machine learning method can capture more complex relationships between the input variables and the response outcome than PLS. As such, ANN might provide better predictions than linear models when such complexities take place in the modeled signal (Felipe et al., 2015).

Thus, the objectives of this study were (1) to evaluate if milk spectra can add information to DMI predictions made using ANN and PLS; (2) to compare ANN- and PLS-based predictions; and (3) to evaluate if WL selection through BN improves model accuracy and precision.

\section{MATERIALS AND METHODS}

\section{Data Set}

One thousand two hundred seventy-nine milk samples from 308 mid-lactating dairy cows (127 \pm 27 DIM) housed either at the Emmons Blaine Dairy Cattle Research Center (Arlington, WI; $\mathrm{n}=248$ cows from 4 experiments) or the US Dairy Forage Research Center (USDFRC; Prairie Du Sac, WI; $\mathrm{n}=60$ cows, from 1 experiment) were collected between 2014 and 2016. All trials were approved by the University of Wisconsin-Madison Institutional Animal Care and Use Committee. Cows were milked twice daily at 0600 and $1500 \mathrm{~h}$ and individual cow milk yield was recorded at each milking. Individual cow milk samples were taken at consecutive a.m. and p.m. milkings, twice weekly. All milk samples were analyzed using MilkoScan FT6000 spectrometers (Foss, Hillerod, Denmark), which generated one spectrum for each milk sample analyzed. The Foss MIR spectrum contains 1,060 data points that represent the absorption of infrared light through the milk sample at WL in the 925 to $5,008 \mathrm{~cm}^{-1}$ range. Wavelengths with less than $1 \%$ coefficient of variation among animals were not used (Figure 1). Therefore, from a total of 1,060 just 361 WL were used.

Individual DMI were periodically recorded from cows housed in a freestall barn equipped with 32 Insentec electronic feeding gates (RIC system; Insentec BV, Marknesse, the Netherlands) or directly measured in a tiestall barn (USDFRC). In addition to DMI, behavior variables were also recorded, which included time spent at the bunk (TB, min/d), total number of visits to the bunk (VB, visits/d), and the visit duration (min/visit for cows housed at the Emmons Blaine Dairy Cattle Research Center; $\mathrm{n}=248$ ). Thus, the trial carried out at USDFRC was not used in the training set of models including behavior parameters. Only daily average visit duration (min/visit) was included in the models because the inclusion of other behavior variables did not improve model predictions in previous tests. Thus, to 
reduce the number of models evaluated in this current study, we included only visit duration. For each milk spectra time point, we recorded DMI, BW, milk yield (MY), fat, protein, lactose, and actual DIM. The prediction equations were developed using the spectrum of the a.m. milk sample, and the individual DMI of the day before the a.m. milk sampling. Body weights of all cows were measured weekly following the a.m. milking.

In experiment 1 , the diet contained, on a DM basis $(\mathrm{g} / \mathrm{kg}): 237$ of alfalfa silage, 288 of corn silage, 122 of high-moisture corn, 47 of cottonseed, and 303 of concentrate mixture with supplemented vitamins and minerals. In the second experiment, the diet contained, on a DM basis $(\mathrm{g} / \mathrm{kg})$ : 343 of corn silage, 172 of alfalfa silage, 114 of high-moisture corn, 47 of cottonseed, and 322 of concentrate mixture with supplemented vitamins and minerals. The third experiment had the following diet on a DM basis $(\mathrm{g} / \mathrm{kg})$ : 282 of corn silage, 235 of alfalfa silage, 115 of high-moisture corn, 47 of cottonseed, and 318 of a concentrate mixture containing supplemented vitamins and minerals. The fourth experiment presented the 6 different diet compositions. All diets contained on a DM basis $(\mathrm{g} / \mathrm{kg})$ : 350 of corn silage, 200 of alfalfa silage, 20 of distiller grains, and 25 of mineral and vitamins. However, they differed in the concentrate composition, which were $(\mathrm{g} / \mathrm{kg}$ of $\mathrm{DM})$ : $\operatorname{diet}(1): 100$ of ground corn, 136 of soybean meal, 105 of molasses, 63 of soyhulls; diet (2): 100 of ground corn, 65 of soybean meal, 105 of molasses, 52 of soyhulls, 82 of SoyPlus (Landus Cooperative, Ames, IA); diet (3): 145 of ground corn, 134 of soybean meal, 52 of molasses, 71 of soyhulls; diet (4): 145 of ground corn, 64 of soybean meal, 51 of molasses, 62 of soyhulls, 80 of soyplus; diet (5): 190 of ground corn, 132 of soybean meal, 77 of soyhulls; diet (6): 190 of ground corn, 63 of soybean meal, 67 of soyhulls, and 79 of soyplus. Finally, the fifth research trial fed the following diet composition $(\mathrm{g} / \mathrm{kg}$ of DM): 415 of corn silage, 124 of alfalfa silage, 100 of high-moisture corn, and 360 of a concentrate mixture containing supplemented vitamins and minerals. Nutrient composition of these diets can be found in Table 1 .

\section{Covariate Sets}

Nine alternative sets of covariates (explanatory variables) were considered to predict DMI of lactating Holstein cows, using either ANN or PLS, as presented in Table 2. The covariate sets considered were as follows: set 1 included MY, BW $\mathrm{BW}^{0.75}$, DIM, and $361 \mathrm{WL}$; set 2 included MY, BW ${ }^{0.75}$, DIM, and 33 WL (WL selected by $\mathrm{BN})$; set 3 included MY, BW ${ }^{0.75}$, DIM, fat, protein, and lactose; set 4 included MY, BW ${ }^{0.75}$, DIM, $33 \mathrm{WL}$, fat, protein, and lactose; set 5 included $\mathrm{MY}, \mathrm{BW}^{0.75}$, DIM, $33 \mathrm{WL}$, and visit duration in the feed bunk; set 6 included MY, DIM, and $33 \mathrm{WL}$; set 7 included MY, $\mathrm{BW}^{0.75}$, and DIM; set-WL included $361 \mathrm{WL}$; and set-

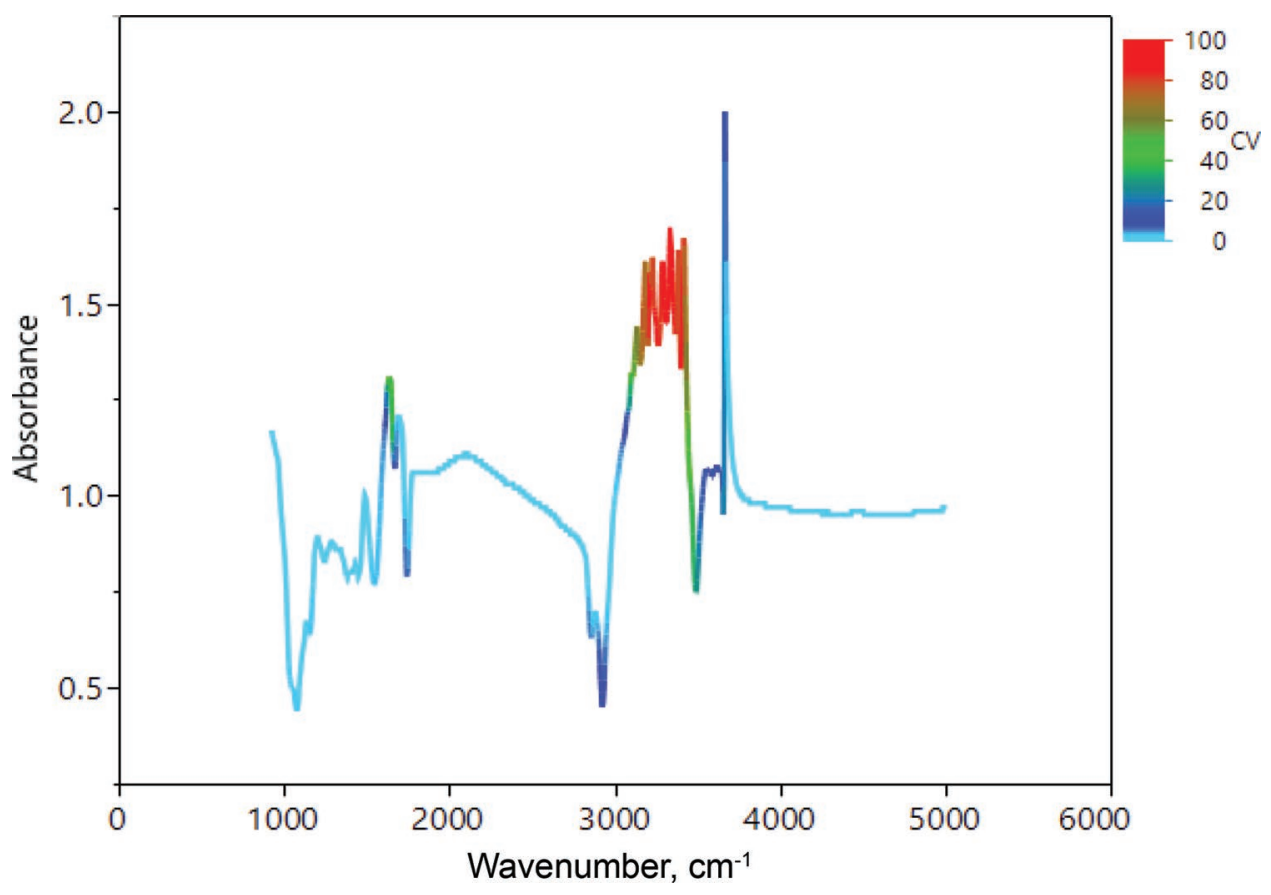

Figure 1. Absorbance and wavenumbers $\left(\mathrm{cm}^{-1}\right)$ from milk Fourier transform mid-infrared spectroscopy $(\mathrm{n}=1,279)$. Color scale of CV from light (blue) to dark (red) representing from lower to higher CV. From 1,060 wavelengths, only 361 presented CV across animals greater than $1 \%$. Color version available online. 
Table 1. Description of the diet composition used in each research trial ${ }^{1}$

Trial 4

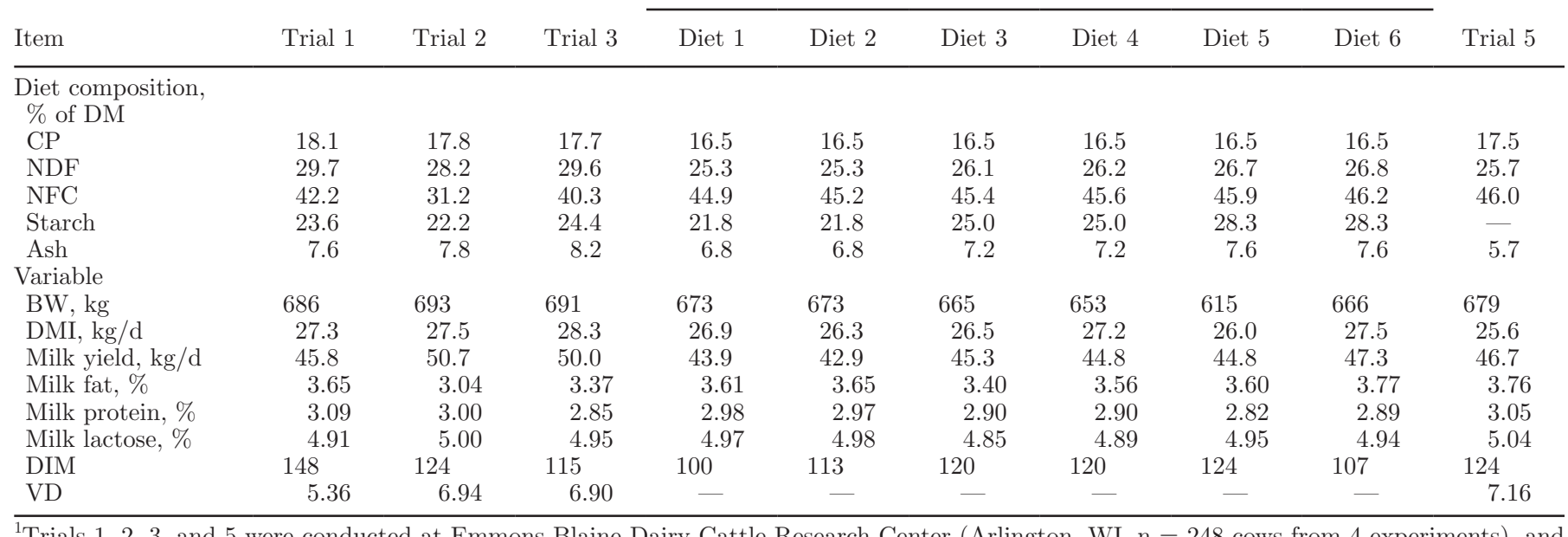

${ }^{1}$ Trials 1, 2, 3, and 5 were conducted at Emmons Blaine Dairy Cattle Research Center (Arlington, WI, $\mathrm{n}=248$ cows from 4 experiments), and trial 4 was conducted at the US Dairy Forage Research Center (Prairie Du Sac, WI, $\mathrm{n}=60$ cows). VD = visit duration (min/visit).

BN included just the WL selected (33 WL) using the MB approach described below.

The prediction quality of ANN and PLS using each set of covariates described above was assessed using 10-fold cross-validation. As an additional step of model testing, from the 5 trials available in this study, 4 were used to train the model, and the remaining trial was used as an external validation set. This approach was repeated 5 times, excluding one trial at a time, which resulted in 5 sub-models for each model (Table 2) and 5 independent validation data sets. Thus, in this current study we will call each of the 5 external validations the subset validation $(\mathbf{S V})$. Results from the $5 \mathrm{SV}$ were combined for an overall model assessment of prediction quality of all 8 sets of covariates, for ANN and PLS.

From the standpoint of model testing, we decided to exclude the whole trial from the data set to more appropriately reproduce a prediction in a completely external data set. We chose one model and compared 2 types of validation: (1) randomly excluding cows from the training data set, or (2) excluding the whole trial from the data set.

\section{Variable Selection-Bayesian Network (Markov Blanket)}

Second, a BN approach was implemented to infer statistical dependences among milk spectra for selection of more informative DMI predictors. The output is a DAG, which is a graphical representation of a probability distribution over a set of variables (Rosa et al., 2016). A DAG consists of a group of nodes (representing variables) connected by directed edges; nodes not directly connected are deemed conditionally independent given at least one subset of nodes in the graph. Felipe et al. (2015) used the concept of MB of a target node (response variable) to define the minimal set of nodes which separates the target node (i.e., makes it conditionally independent in the joint distribution) from all the others.

Table 2. Description of the different sets of covariates used to predict DMI using milk yield (MY), BW ${ }^{0.75}$, DIM, visit duration at the feed bunk (VD), milk components (fat, protein, and lactose), and milk Fourier transform mid-infrared spectra for partial least squares (PLS) and artificial neural networks (ANN)

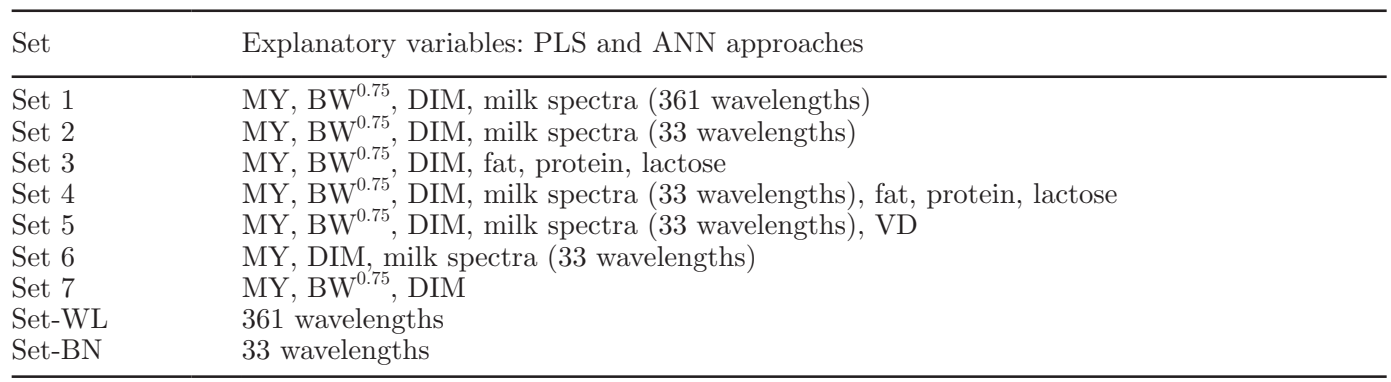


The package bnlearn (Scutari, 2010) in R ( $\mathrm{R}$ Core Team, 2016) was used to fit the BN model, and the function learn.mb was used to find the MB of the DMI using all WL in the milk spectra as potential covariates $(\mathrm{n}=1,060$, Figure 1$)$. In this present study, a grow-shrink algorithm was used to implement the BN learning structure.

\section{Partial Least Squares}

A partial least square regression (PROC PLS; SAS 9.4, SAS Institute Inc., Cary, NC) was used to predict DMI from MY, BW, DIM, and milk spectra data. The PLS extracts successive linear combinations of the predictors, called factors, which address both explaining response variation and explaining predictor variation.

The number of factors retained in the model was determined using a $10 \mathrm{k}$-fold cross-validation, with the predicted residual sum of squares (PRESS) statistics calculated. A model with a smaller PRESS value is preferred. However, because the difference in PRESS value between models with different numbers of factors is so small, a statistical model comparison suggested by van der Voet (1994) was used to test whether the difference was significant. The test was performed by using the CVTEST option in SAS. The description of the developed PLS models can be found in Table 3 .

\section{Artificial Neural Network}

Artificial neural networks were developed using the open-source software for big data analysis called $\mathrm{H} 2 \mathrm{O}$ (https://cran.r-project.org/web/packages/h2o/h2o .pdf), which can be used through the statistical software $\mathrm{R}$ ( $\mathrm{R}$ Core Team, 2016). The H2O package is used for exploring and analyzing data sets held in cloud computing systems and in the Apache Hadoop distributed file system, as well as in the conventional operating systems Linux, macOS, and Microsoft Windows.
Usually, algorithms used in machine learning have a set of tuning parameters that affect how the learning algorithm fits to the data. For instance, in the deep learning neural networks (DLNN), the number of layers, the number of neurons in each layer, learning rate, and other features are tuning parameters called hyperparameters. Because many parameters can affect model accuracy, hyperparameter tuning is essential for DLNN. Thus, to select the best combination of hyperparameters we performed a grid search using the function h2o.grid and specifying the range of hyperparameters as search criteria. Random combinations of all hyperparameters defined in the grid search [6 activation functions, 16 hidden layers, 2 input dropout ratios, 3 levels of lasso regularization $(\mathbf{L} \mathbf{1})$, and 3 levels of ridge regularization (L2, Table 4)] were selected to build the models. To reduce the time training the models, early stopping was set based on 3 parameters: maximum running time, maximum number of models, and stopping metrics (root mean square error). More detailed information about the defined hyperparameters and search criteria can be found in Table 4 .

The defined hyperparameters are the same for all sub-models within a model. To accomplish that, we selected from the ranking list given by the grid search the top 5 models, and we investigated them to find the same hyperparameters for sub-models from the same model (i.e., the same number of neurons, layers, activation function, and so on, for all 5 sub-models from one specific model).

After the grid search, the hyperparameters for the best model were chosen for each model presented in Table 2. Each model with its respective ANN structures was trained through $10 \mathrm{k}$-fold cross-validation and tested using SV. The function h2o.deeplearning was used to train the models. The ADADELTA was the adaptive learning rate algorithm used in all ANN (Zeiler, 2012). This learning rate automatically combines the benefits of learning rate annealing and momentum training to

Table 3. Models developed based on the factors defined by predicted residual sum of squares in partial least squares (PLS)

\begin{tabular}{|c|c|c|c|c|c|c|c|c|c|}
\hline \multirow[b]{2}{*}{ Item } & \multicolumn{9}{|c|}{ Covariate $\operatorname{set}^{1}:$ PLS } \\
\hline & $\mathrm{S} 1$ & $\mathrm{~S} 2$ & S3 & $\mathrm{S} 4$ & $\mathrm{~S} 5$ & S6 & S7 & Set-WL & Set-BN \\
\hline PLS factors & 9 & 6 & 5 & 7 & 5 & 6 & 2 & 4 & 8 \\
\hline \multicolumn{10}{|l|}{ Training set } \\
\hline \multicolumn{10}{|l|}{ 10-fold cross-validation ${ }^{2}$} \\
\hline RMSE, kg/d & 1.69 & 2.14 & 2.32 & 2.04 & 2.14 & 2.51 & 2.44 & 3.47 & 3.29 \\
\hline $\mathrm{R}^{2}$ & 0.81 & 0.71 & 0.67 & 0.73 & 0.70 & 0.59 & 0.62 & 0.22 & 0.31 \\
\hline
\end{tabular}

${ }^{1}$ Sets of explanatory variables used for prediction in each model: set $1=$ milk yield (MY), BW ${ }^{0.75}$, DIM, and 361 wavelengths (WL); set $2=$ MY, $\mathrm{BW}^{0.75}$, DIM and $33 \mathrm{WL}$; set $3=\mathrm{MY}, \mathrm{BW}^{0.75}$, DIM, fat, protein, and lactose; set $4=\mathrm{MY}$, BW ${ }^{0.75}$, DIM and 33 WL, fat, protein, and lactose; set $5=\mathrm{MY}, \mathrm{BW}^{0.75}$, DIM, $33 \mathrm{WL}$, and visit duration in the feed bunk; set $6=\mathrm{MY}$, DIM, and 33 WL; set $7=\mathrm{MY}$, BW ${ }^{0.75}$, and DIM; set-WL $=361 \mathrm{WL}$; and set-BN $=33 \mathrm{WL}$.

${ }^{2}$ Root mean square error (RMSE) and $\mathrm{R}^{2}$ from training set 10-fold cross-validation. 
avoid slow convergence. The data set was also preprocessed for compatibility with the activation functions. Because the activation function does not generally map into the real numbers, we standardized the data set to a mean of 0 and a variance of 1 . The data set was standardized again after network propagation, which can compute more precisely errors in this standardized space, in comparison to the raw feature space. The description of the developed ANN can be found in Table 5 .

\section{External Model Validation and Assessment}

The model evaluation was conducted as described by Tedeschi (2006). The precision of the developed equations was evaluated using the coefficient of determination $\left(\mathbf{R}^{2}\right)$ between the predicted and observed values. The accuracy was calculated based on the concordance correlation coefficient (CCC; Lin, 1989), mean bias, and root of mean square error prediction (RMSEP).

The RMSEP equation consists of the square root of mean square error prediction (MSEP), where MSEP is the sum of the squared difference between observed values and model-predicted values divided by the number of data points (n; Bibby and Toutenburg, 1977). The MSEP was decomposed into mean bias, slope bias, and random errors (Theil, 1961) to provide an indication of the adequacy of the model for prediction. The mean bias represents the error in the central tendency (to assess whether the predicted values are over- or underpredicted), the slope bias represents errors due to regression, and the random component is the unexplained variance that cannot be accounted for by the linear regression (Tedeschi, 2006). Model evaluations were performed using the software Model Evaluation System (MES, College Station, TX; http://nutritionmodels .com/) as described by Tedeschi (2006).

\section{RESULTS AND DISCUSSION}

The use of ANN improved the accuracy and precision of DMI predictions for covariate sets including $33 \mathrm{WL}$ (sets 2, 5, 6, and BN; RMSEP $=2.36,2.15,2.78$, and $2.78 \mathrm{~kg} / \mathrm{d}$, and $\mathrm{R}^{2}=0.67,0.70,0.52$, and 0.17 , respectively; Table 6) compared with PLS (RMSEP $=2.65$, $2.70,2.93$, and $3.80 \mathrm{~kg} / \mathrm{d}$, and $\mathrm{R}^{2}=0.56,0.54,0.46$, and 0.13 , respectively; Table 6). Partial least squares was designed as an intersection between linear modeling and principal component analysis: a linear model capable of determining which predictor variables were best related to the response variable and appropriately dealing with multicollinearity (Wold, 2004). However,

Table 4. Hyperparameters and search criteria used in the grid search of artificial neural network (ANN) ${ }^{1}$

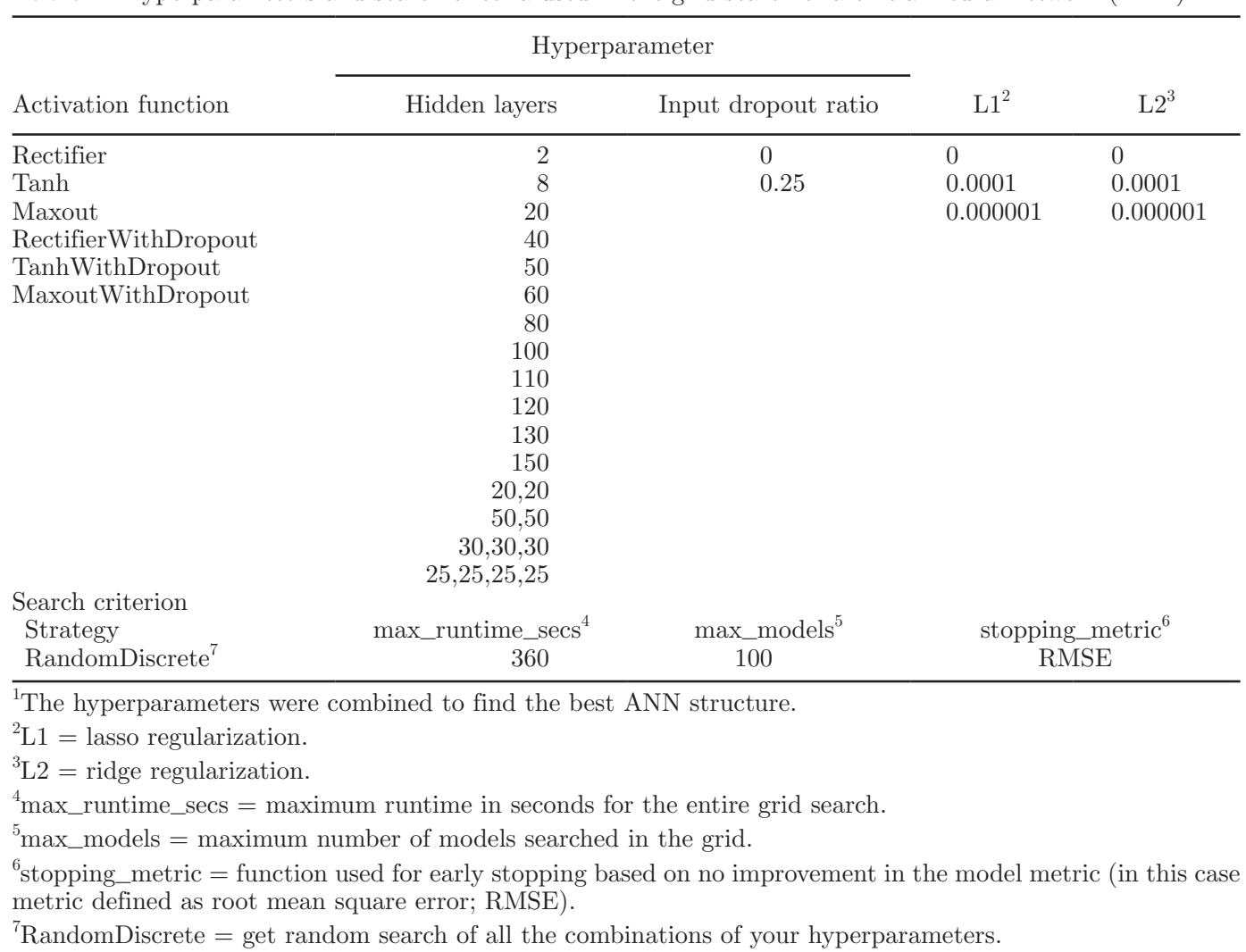


DÓREA ET AL.

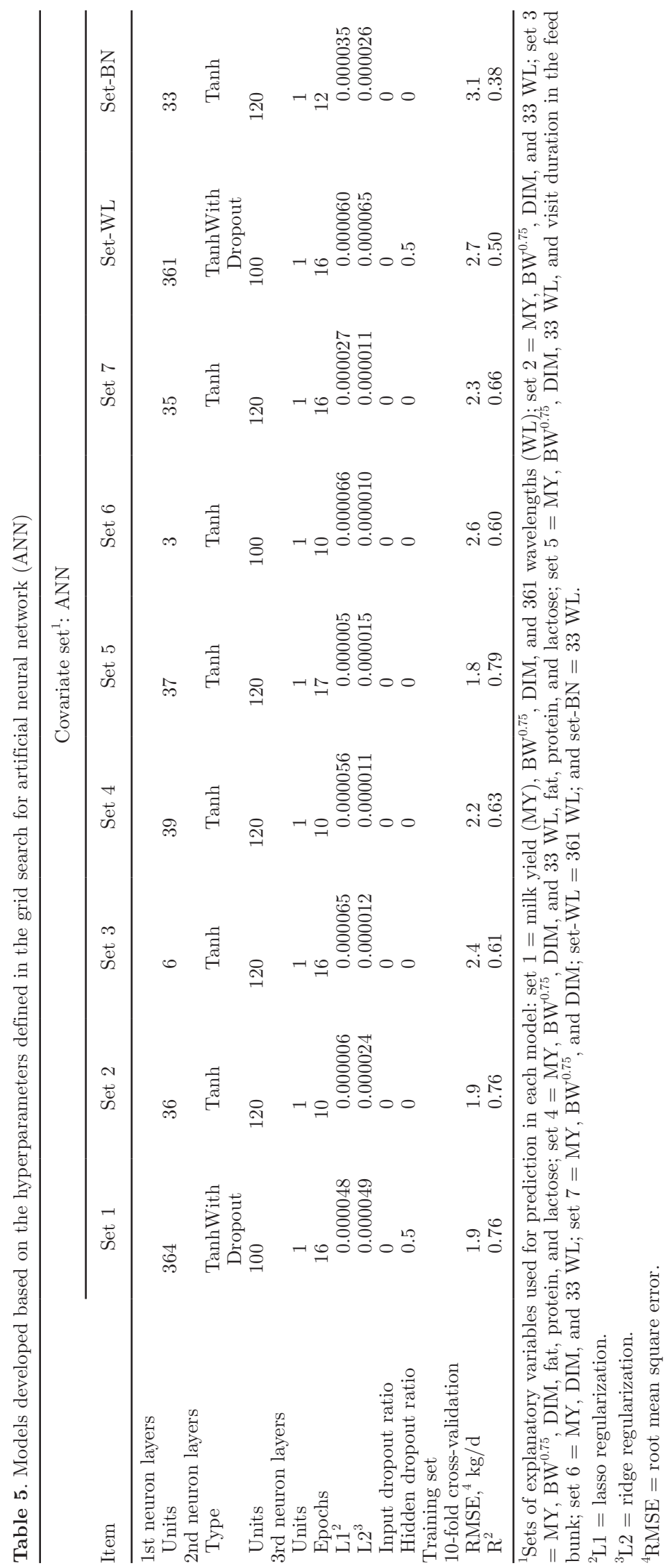


Table 6. Parameter evaluation of DMI predictions from different models using cow variables [milk yield (MY), BW, and DIM], feeding behavior (visit duration), and milk spectra

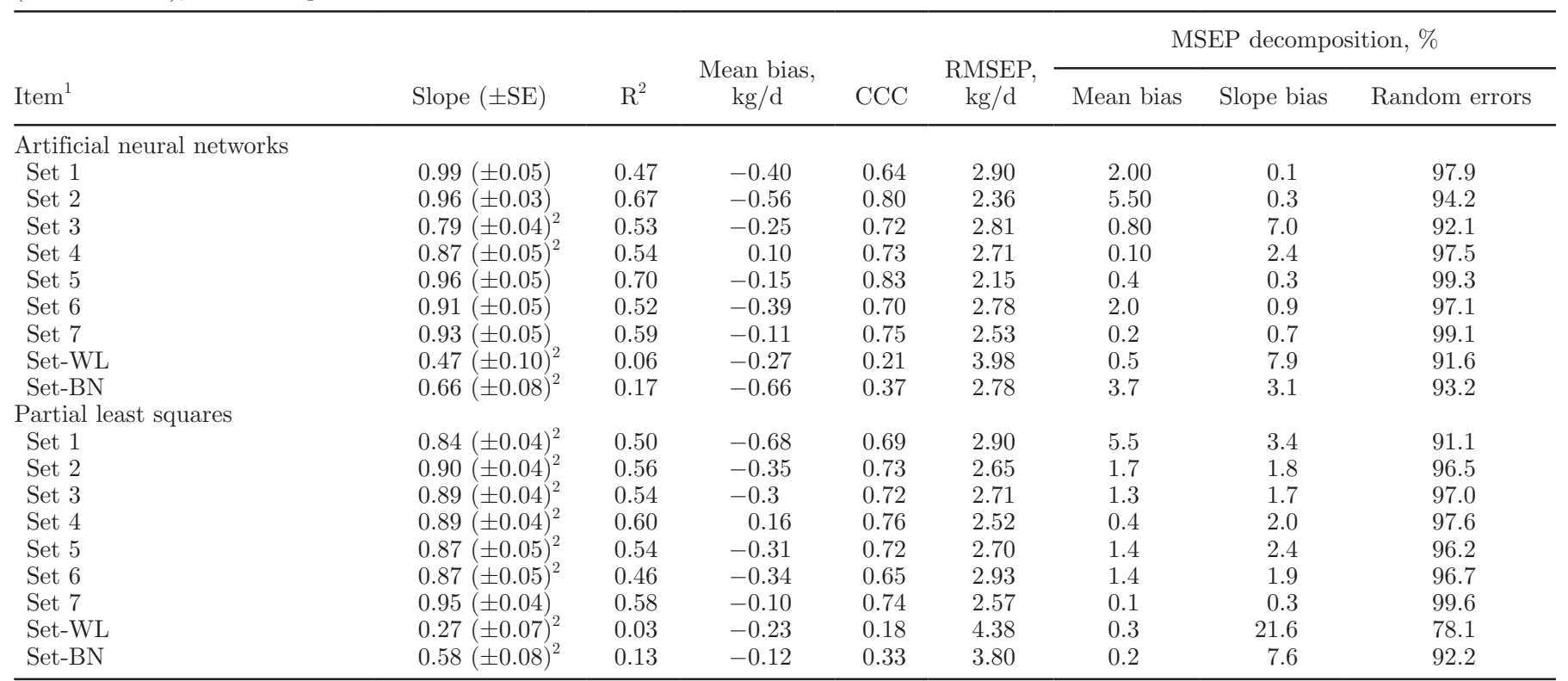

${ }^{1}$ Sets of explanatory variables used for prediction in each model: set $1=\mathrm{MY}, \mathrm{BW}^{0.75}$, DIM, and 361 wavelengths $(\mathrm{WL}) ;$ set $2=\mathrm{MY}^{0}, \mathrm{BW}^{0.75}$, $\mathrm{DIM}$ and $33 \mathrm{WL}$; set $3=\mathrm{MY}, \mathrm{BW}^{0.75}$. DIM, fat, protein, and lactose; set $4=\mathrm{MY}, \mathrm{BW}^{0.75}$, DIM, and $33 \mathrm{WL}$, fat, protein, and lactose; set $5=$ MY, BW ${ }^{0.75}$, DIM, $33 \mathrm{WL}$, and visit duration in the feed bunk; set $6=\mathrm{MY}$, DIM, and $33 \mathrm{WL}$; set $7=\mathrm{MY}$, BW ${ }^{0.75}$, and DIM; set-WL = 361 $\mathrm{WL}$; and set-BN $=33 \mathrm{WL}$

${ }^{2}$ Slope test $(b \neq 1), P<0.05$.

PLS is constrained by the linearity assumption, and that is where ANN has a distinct advantage as it does not require linearity. Thus, to more effectively model potentially nonlinear relationships between input and output variables, machine learning approaches are a powerful and flexible choice, and as this current study and others (Perai et al., 2010; Lin et al., 2012) demonstrate, it can provide superior predictions compared with PLS. The superiority of ANN over PLS, as found in this study, indicates that the relationship between DMI and some WL may be nonlinear.

Interestingly, the ANN did not improve the accuracy and precision of set $1\left(\mathrm{ANN}: \mathrm{R}^{2}=0.47, \mathrm{RMSEP}=2.9\right.$ $\mathrm{kg} / \mathrm{d}$; PLS: $\mathrm{R}^{2}=0.50, \mathrm{RMSEP}=2.9 \mathrm{~kg} / \mathrm{d}$; Table 6 ), which included milk yield, BW, DIM, and $361 \mathrm{WL}$. One possible reason for this result is that ANN can capture features that are noninformative. Thus, if most of the WL are noninformative (or noise) in the spectra, the ANN could yield poorer predictions compared with the PLS approach. One indication of noninformative inputs in the set 1 is the activation function given by the grid search as being "TanhWithDropout" (Table 5). The activation function "Tanh" coupled with "Dropout" is usually used to prevent overfitting and to provide a way to approximately combine exponentially many ANN architectures efficiently (Srivastava et al., 2014). By applying the dropout strategy, the algorithm drops part of the units out, which means it temporarily removes the unit and all incoming and outgoing connections from the network (Srivastava et al., 2014). The input dropout ratio used in set 1 and set-WL was 0.5 (Table 5), which means $50 \%$ of dropout units. However, it was not enough to improve the model prediction. Another adopted strategy to reduce overfitting in the ANN was the use of lasso (L1) and ridge (L2) regularization. In the ANN the L1 regularization constrains the absolute value of the weights, leading to a sparse vector during optimization (i.e., very close to zero), which has an effect on dropout (Hastie et al., 2001; Zou and Hastie, 2005). The L2 regularization constrains the sum of the squared weights, also called weight decay, which is analogous to ridge regression used for linear models (Hastie et al., 2001; Zou and Hastie, 2005). In the present study, we combined both penalty methods (L1 and L2) as way to control overfitting (Table 5). All other ANN presented "Tanh" as the activation function with the number of units (neurons) in the hidden layers varying from 100 to 120 (Table 5).

Although ANN architectures with more than one hidden layer (DLNN) were included in the grid search, none of them were selected (Table 5) in the top 100 listed models. Deep neural networks, which contain multiple nonlinear hidden layers, are very powerful models that can learn very complicated relationships 
between input and output (Srivastava et al., 2014). However, in training data sets with limited amount of data, noise in the data may be captured by the model so that apparently complex relationships appear in the training set, but not in an independent test data set (Srivastava et al., 2014). Although our data set involves some level of complexity due to potential nonlinear relationships between many WL and DMI, the size of the data set probably prevented complex models from being selected as the top models listed in the grid search.

Studies applying ANN as a tool to predict calving (Borchers et al., 2017), subclinical ketosis (Ehret et al., 2015), and estrus (Dolecheck et al., 2016) have been published recently. However, most of the studies found in the animal and dairy science literature do not describe how the ANN architecture was defined. The determination of an ideal ANN architecture is the most challenging task when this technique is employed because it differs for each data set (Albelwi and Mahmood, 2017). Nonetheless, there are always many possible combinations of hyperparameters that result in specific network architectures, which can dramatically affect the quality of the prediction. Thus, conclusions could be misled regarding prediction capability, mainly when studies compare different models or machine learning techniques.

The use of $\mathrm{BN}(\mathrm{MB})$ to reduce the data dimension from 361 to $33 \mathrm{WL}$, combined with ANN, was a powerful strategy to improve accuracy and precision in the tested models. This observation agrees with work by Felipe et al. (2015), who previously used this combination of BN and ANN to model egg traits in chickens. It should be kept in mind that although machine learning methods do not necessarily provide a biological explanation for the relationship between predictor and response variables, the pattern recognition and predictive capabilities are key factors to conduct analysis of large data sets, namely to predict response variables accurately and precisely.

In the PLS models, we did not observe any improvement from including individual WL (33 WL, set 2) compared with milk components (fat, protein, and lactose; set 3 ) as they presented similar $R^{2}(0.56$ and 0.54 , respectively), CCC (0.73 and 0.72 , respectively), and RMSEP (2.65 and $2.71 \mathrm{~kg} / \mathrm{d}$, respectively; Table $6)$. This result agrees with findings from Shetty et al. (2017) who were unable to improve PLS models with the addition of individual WL in comparison with milk components. It is important to point out that milk fat, protein, and lactose are not determined by wet chemistry, but predicted based on milk infrared spectroscopy. However, when a machine learning technique was employed, in this case ANN, the model prediction with the inclusion of $33 \mathrm{WL}$ was improved over the model containing milk components, in our case concentration of fat, protein, and lactose in milk $(\mathrm{CCC}=0.80$ vs. $0.72 ; \mathrm{R}^{2}=0.67$ vs. $0.53 ;$ RMSEP 2.36 vs. $2.81 \mathrm{~kg} / \mathrm{d}$; Table 6). These results suggest that useful information is present in the raw spectra (individual WL), and that its extraction or interpretation can be limited when linear models are used. Other chemical components in milk may play an important role to improve quality of DMI predictions when raw milk spectra are used. However, other molecules present in milk that are not well known or unable to be predicted could be extracted by using raw milk spectra, since an adequate approach to extract the information is used.

When raw milk spectra were combined with milk components (set 4), the accuracy and precision of PLS models were increased $\left(\mathrm{CCC}=0.76, \mathrm{R}^{2}=0.60\right.$, RMSEP $=2.52 \mathrm{~kg} / \mathrm{d}$ ) compared with set of covariates including only milk components (set 3) or raw milk spectra (set 2). However, the set of covariates without milk components (set 2) used as input in ANN prediction presented greater accuracy and precision $(\mathrm{CCC}=$ $0.80, \mathrm{R}^{2}=0.67, \mathrm{RMSEP}=2.36 \mathrm{~kg} / \mathrm{d}$, Table 6 ). This result reinforces the potential of ANN to extract useful information hidden in the raw spectra.

Our study demonstrated that even when utilizing ANN, a set of covariates including only WL is insufficient to generate useful predictions (set-BN: $\mathrm{R}^{2}=0.17$ and 0.13 for ANN and PLS, respectively). McParland et al. (2014) predicted effective energy intake with PLS using only milk spectra from AM and PM milk samples and observed an $R^{2}=0.30$. However, when they included milk yield in the model, the $\mathrm{R}^{2}$ improved to 0.49 , which is similar to set 6 with DIM, MY, and $33 \mathrm{WL}\left(\mathrm{R}^{2}\right.$ of 0.46 and 0.50 for PLS and ANN, respectively). This indicates that whereas spectra are useful, other cow variables that are readily available on farm can further improve models and should be included. It is important to point out that the majority of the animals used by McParland et al. (2014) were grazing cows, and that the DMI was estimated by n-alkanes.

The inclusion of a behavior parameter average (visit duration) in ANN set 5 resulted in the best predictions compared with the other sets $\left(\mathrm{R}^{2}=0.70, \mathrm{CCC}\right.$ $=0.83$, RMSEP $=2.15 \mathrm{~kg} / \mathrm{d}$ ). The average visit duration is the total time spent in the bunk divided by the number of visits per day. Studies have reported a good correlation between number of visits, eating time, and visit duration with DMI in lactating dairy cows (Dado and Allen, 1994; Schwartzkopf-Genswein et al., 2002). The amount of data generated by livestock systems has increased over the years (Wolfert et al., 2017). The possibility of integrating data from multiple sources, such as DHI companies, which provide herd milk test, animal record systems (software containing the cow 
identification, milk yield, DIM, BW, and so on), and data from sensors, such as weather, cow's temperature, and feeding behavior (Dolecheck et al., 2016; Borchers et al., 2017) could be a powerful tool to improve dairy farm management. In this present study, set 5 included milk yield, BW, DIM, milk spectra (33 WL), and feeding behavior (visit duration), which are all possible to integrate on dairy farms with those data sources. A fast and accurate measurement of cow-level feed intake would greatly benefit dairy production systems, which require this information to monitor cow performance at the farm. However, it is important to point out that at least 2 milk samples per cow should be taken to have greater precision and accuracy of the predictions. When set 2 was used with ANN to predict DMI from single milk samples, the CCC, $\mathrm{R}^{2}$, and RMSEP were 0.57 , 0.36 , and $3.93 \mathrm{~kg} / \mathrm{d}$, respectively. These results indicate worse DMI prediction when single milk samples are used, compared with results obtained with the same set of covariates (set 2) when using averaged milk samples (Table 6).

When validating models with an external data set, it is common to find studies that exclude cows or some records of each cow from the training data set (McParland et al., 2014; Klaffenböck et al., 2017; Shetty et al., 2017) to create an independent data set for validation. Shetty et al. (2017) compared a model validation by using external data sets determined in 2 different ways. One data set was created by random exclusion of cows (and all records from that cow) from the training set, and the second data set was created by randomly excluding records, not necessarily the whole-cow information, from the training data sets. In both cases the validation set was $20 \%$ of the training data set. These authors concluded that when the cow record is not entirely excluded from the training set the model prediction is more optimistic compared with excluding the entire cow from the training set. In other words, there is an inflation in the model predictions by keeping records from the same cow in both data sets (training and validation). In the present study, we further investigated this topic hypothesizing that even excluding a cow from the training data set, conditions related to external factors such as weather, diet, season, management, and others would inflate the prediction quality. Thus, we decided to compare the validation using the exclusion of the entire cow record from the training set (excluding cow validation, ECV), as recommended by Shetty et al. (2017), with the exclusion of an entire trial from the training data set (excluding trial validation, ETV) for a given scenario (set 2). It is important to point out that cows were unique in each trial, meaning that no cows were repeated across trials. Hence, by excluding a trial (as a validation set), we definitely excluded the entire cow information. The use of ECV increased the $\mathrm{R}^{2}$ by $21 \%$ and $7 \%$ for PLS and ANN, respectively (Table 7 ). In addition, RMSEP and mean bias were lower for ECV compared with ETV, for both PLS and ANN. Clearly, an inflation in the PLS prediction ability occurred when cows from the same trial were kept in both the training and validation data set. Our proposal to validate the models by excluding the entire set, in this case a whole trial, is closer to reality because the developed models are supposed to be used to predict new data sets coming from farms or herds with different diets, weather, and management practices. Other authors have realized the importance of independent data sets for validation of models (McParland et al., 2011), but not all have yet recognized that it is vital to determining if a model can be used effectively by other parties.

\section{CONCLUSIONS}

The addition of WL selected through BN to models containing cow variables improved the accuracy and pre-

Table 7. Parameters evaluation of DMI predictions using set $2^{1}$ and 2 different validation methods ${ }^{2}$

\begin{tabular}{|c|c|c|c|c|c|c|c|c|}
\hline Scenario & Slope $( \pm \mathrm{SE})$ & $\mathrm{R}^{2}$ & $\begin{array}{l}\text { Mean bias, } \\
\mathrm{kg} / \mathrm{d}\end{array}$ & $\mathrm{CCC}$ & $\begin{array}{c}\mathrm{RMSEP} \\
\mathrm{kg} / \mathrm{d}\end{array}$ & \multicolumn{3}{|c|}{ MSEP decomposition, $\%$} \\
\hline Set 2: ANN & $0.96( \pm 0.03)$ & 0.67 & -0.56 & 0.80 & 2.36 & 5.5 & 0.3 & 94.2 \\
\hline \multicolumn{9}{|l|}{ ECV } \\
\hline Set 2: PLS & $1.02( \pm 0.05)$ & 0.68 & 0.21 & 0.80 & 2.20 & 0.9 & 0.1 & 99.0 \\
\hline
\end{tabular}

${ }^{1}$ Set 2 = milk yield, BW ${ }^{0.75}$, DIM, and 33 wavelengths.

${ }^{2}$ Validation methods = ETV: validation was performed by excluding one entire trial and validating the models in each excluded trial; ECV: validation was performed by randomly excluding cows from the training set to be used as validation set. RMSEP $=$ root of mean square error prediction; MSEP = sum of the squared difference between observed values and model-predicted values divided by the number of data points. PLS $=$ partial least squares; ANN = artificial neural network.

${ }^{3}$ Slope test $(b \neq 1), P<0.05$. 
cision of DMI predictions in lactating dairy cows when ANN was used. The improvement of ANN predictive ability over PLS indicates possible nonlinear relationships between DMI and individual WL. The improved performance of models including raw spectra compared with milk components (fat, protein, and lactose) in ANN indicates that other unknown compounds may play an important role in improving prediction quality. In addition, the benefit of including raw milk spectra in ANN but not in PLS reinforces the use of ANN as a superior approach to extract useful information from compounds hidden in milk. Thus, future work should go beyond the use of PLS and principal components when analyzing data before concluding that a relationship does not exist or is not accurate and precise enough to be useful. In both ANN and PLS approaches, the use of a ECV inflated model predictions, suggesting the presence of carryover effects from cows from the same trial remaining in both training and validation data sets. Future studies with milk spectra utilizing external validation should be carefully conducted to simulate a scenario similar to independent farms.

\section{ACKNOWLEDGMENTS}

This research was partially funded by USDA National Institute of Food and Agriculture (Washington, DC; grant 2011-68004-30340).

\section{REFERENCES}

Agricultural Research Council (ARC). 1980. The Nutrient Requirements of Ruminant Livestock, Technical Review. CABI, Farnham Roval, UK.

Albelwi, S., and A. Mahmood. 2017. A Framework for Designing the Architectures of Deep Convolutional Neural Networks. Entropy (Basel) 19:242-262. https://doi.org/10.3390/e19060242.

Bibby, J., and H. Toutenburg. 1977. Prediction and Improved Estimation in Linear Models. John Wiley and Sons, Berlin, Germany.

Bishop, C. M. 2006. Pattern Recognition and Machine Learning. Springer, New York, NY.

Borchers, M. R., Y. M. Chang, K. L. Proudfoot, B. A. Wadsworth, A. E. Stone, and J. M. Bewley. 2017. Machine-learning-based calving prediction from activity, lying, and ruminating behaviors in dairy cattle. J. Dairy Sci. 100:5664-5674.

Coppa, M., A. Ferlay, C. Leroux, M. Jestin, Y. Chilliard, B. Martin, and D. Andueza. 2010. Prediction of milk fatty acid composition by near infrared reflectance spectroscopy. Int. Dairy J. 20:182-189.

Dado, R. G., and M. S. Allen. 1994. Variation in and relationships among feeding, chewing, and drinking variables for lactating dairy cows. J. Dairy Sci. 77:132-144.

Dagnachew, B. S., T. H. E. Meuwissen, and T. Ådnøy. 2013. Genetic components of milk Fourier-transform infrared spectra used to predict breeding values for milk composition and quality traits in dairy goats. J. Dairy Sci. 96:5933-5942. https://doi.org/10.3168/ jds.2012-6068.

Dolecheck, K. A., W. J. Silvia, G. Heersche Jr., C. L. Wood, K. J. McQuerry, and J. M. Bewley. 2016. A comparison of timed artificial insemination and automated activity monitoring with hormone intervention in 3 commercial dairy herds. J. Dairy Sci. 99:1506-1514. https://doi.org/10.3168/jds.2015-9914.
Dórea, J. R. R., E. A. French, and L. E. Armentano. 2017. Use of milk fatty acids to estimate plasma nonesterified fatty acid concentrations as an indicator of animal energy balance. J. Dairy Sci. 100:6164-6176.

Ehret, A., D. Hochstuhl, N. Krattenmacher, J. Tetens, M. S. Klein, W. Gronwald, and G. Thaller. 2015. Use of genomic and metabolic information as well as milk performance records for prediction of subclinical ketosis risk via artificial neural networks. J. Dairy Sci. 98:322-329.

Eskildsen, C. E., M. A. Rasmussen, S. B. Engelsen, L. B. Larsen, N. A. Poulsen, and T. Skov. 2014. Quantification of individual fatty acids in bovine milk by infrared spectroscopy and chemometrics: Understanding predictions of highly collinear reference variables. J. Dairy Sci. 97:7940-7951.

Felipe, V. P. S., M. A. Silva, B. D. Valente, and G. J. M. Rosa. 2015. Using multiple regression, Bayesian networks and artificial neural networks for prediction of total egg production in European quails based on earlier expressed phenotypes. Poult. Sci. 94:772-780.

Fox, D., L. O. Tedeschi, T. Tylutki, J. Russell, M. Van Amburgh, L. Chase, A. Pell, and T. Overton. 2004. The Cornell Net Carbohydrate and Protein System model for evaluating herd nutrition and nutrient excretion. Anim. Feed Sci. Technol. 112:29-78. https:// doi.org/10.1016/j.anifeedsci.2003.10.006

Gianola, D., H. Okut, K. A. Weigel, and G. J. M. Rosa. 2011. Predicting complex quantitative traits with Bayesian neural networks: A case study with Jersey cows and wheat. BMC Genet. 12:87-99. https://doi.org/10.1186/1471-2156-12-87.

Hastie, T., R. Tibshirani, and J. Friedman. 2001. The Elements of Statistical Learning: Data Mining, Inference and Prediction. Springer, New York, NY.

Klaffenböck, M., A. Steinwidder, C. Fasching, G. Terler, L. Gruber, G. Mészáros, and J. Sölkner. 2017. The use of mid-infrared spectrometry to estimate the ration composition of lactating dairy cows. J. Dairy Sci. 100:5411-5421. https://doi.org/10.3168/jds.2016-12189.

Lin, L. I. 1989. A concordance correlation coefficient to evaluate reproducibility. Biometrics 45:255-268.

Lin, M. I. B., W. A. Groves, A. Freivalds, E. G. Lee, and M. Harper. 2012. Comparison of artificial neural network (ANN) and partial least squares (PLS) regression models for predicting respiratory ventilation: an exploratory story. Eur. J. Appl. Physiol. 112:16031611. https://doi.org/10.1007/s00421-011-2118-6

McParland, S., G. Banos, E. Wall, M. P. Coffey, H. Soyeurt, R. F. Veerkamp, and D. P. Berry. 2011. The use of mid-infrared spectrometry to predict body energy status of Holstein cows. J. Dairy Sci. 94:3651-3661.

McParland, S., E. Lewis, E. Kennedy, S. G. Moore, B. McCarthy, M. O'Donovan, S. T. Butler, J. E. Pryce, and D. P. Berry. 2014. Mid-infrared spectrometry of milk as a predictor of energy intake and efficiency in lactating dairy cows. J. Dairy Sci. 97:5863-5871.

NRC. 2001. Nutrient Requirements of Dairy Cattle. 7th rev. ed. Natl. Acad. Press, Washington, DC.

Perai, A. H., H. Nassiri Moghaddam, S. Asadpour, J. Bahrampour, and G. Mansoori. 2010. A comparison of artificial neural networks with other statistical approaches for the prediction of true metabolizable energy of meat and bone meal. Poult. Sci. 89:1562-1568. https://doi.org/10.3382/ps.2010-00639.

R Core Team. 2016. R: A language and environment for statistical computing R Foundation for Statistical Computing, Vienna, Austria. https://www.R-project.org/.

Rinnan, Å., M. Andersson, C. Ridder, and S. B. Engelsen. 2014. Recursive weighted partial least squares (rPLS): An efficient variable selection method using PLS. J. Chemometr. 28:439-447. https:// doi.org/10.1002/cem.2582.

Rosa, G. J. M., V. P. S. Felipe, and F. Peñagaricano. 2016. Applications of graphical models in quantitative genetics and genomics. Pages 95-116 in Systems Biology in Animal Production and Health, Vol. 1. H. N. Kadarmideen, ed. Springer, New York, NY. https://doi.org/10.1007/978-3-319-43335-6_5.

Schwartzkopf-Genswein, K. S., S. Atwood, and T. A. McAllister. 2002. Relationships between bunk attendance, intake and performance 
of steers and heifers on varying feeding regimes. Appl. Anim. Behav. Sci. 76:179-188.

Scutari, M. 2010. Learning Bayesian Networks with the bnlearn R Package. J. Stat. Softw. 35:1-22.

Shetty, N., P. Løvendahl, M. Lund, and A. Buitenhuis. 2017. Prediction and validation of residual feed intake and dry matter intake in Danish lactating dairy cows using mid-infrared spectroscopy of milk. J. Dairy Sci. 100:253-264.

Soyeurt, H., C. Bastin, F. G. Colinet, V. M. R. Arnould, D. P. Berry, E. Wall, F. Dehareng, H. N. Nguyen, P. Dardenne, J. Schefers, J. Vandenplas, K. Weigel, M. Coffey, L. Theron, J. Detilleux, E. Reding, N. Gengler, and S. McParland. 2012. Mid-infrared prediction of lactoferrin content in bovine milk: Potential indicator of mastitis. Animal 6:1830-1838.

Soyeurt, H., P. Dardenne, F. Dehareng, G. Lognay, D. Veselko, M. Marlier, C. Bertozzi, P. Mayeres, and N. Gengler. 2006. Estimating fatty acid content in cow milk using mid-infrared spectrometry. J. Dairy Sci. 89:3690-3695.

Soyeurt, H., F. Dehareng, N. Gengler, S. McParland, E. Wall, D. P. Berry, M. Coffey, and P. Dardenne. 2011. Mid-infrared prediction of bovine milk fatty acids across multiple breeds, production systems, and countries. J. Dairy Sci. 94:1657-1667.

Srivastava, N., G. Hinton, A. Krizhevsky, I. Sutskever, and R. Salakhutdinov. 2014. Dropout: A simple way to prevent neural networks from overfitting. J. Mach. Learn. Res. 15:1929-1958.
Tedeschi, L. O. 2006. Assessment of the adequacy of mathematical models. Agric. Syst. 89:225-247. https://doi.org/10.1016/j.agsy .2005.11.004

Theil, H. 1961. Economic forecasts and policy. Pages 6-48 in Contributions to Economic Analysis. R. Strotz, J. Tinbergen, P. Verdoorn, and H. J. Witteveen, ed. North-Holland Publishing Company, Amsterdam, the Netherlands.

van der Voet, H. 1994. Comparing the predictive accuracy of models using a simple randomization test. Chemometr. Intell.Lab. $25: 313-323$.

Vanlierde, A., M. L. Vanrobays, N. Gengler, P. Dardenne, E. Froidmont, H. Soyeurt, S. McParland, E. Lewis, M. H. Deighton, M. Mathot, and F. Dehareng. 2016. Milk mid-infrared spectra enable prediction of lactation-stage-dependent methane emissions of dairy cattle within routine population-scale milk recording schemes. Anim. Prod. Sci. 56:258-264.

Wold, H. 2004. Partial Least Squares. Pages 581-591 in Encyclopedia of Statistical Sciences. John Wiley \& Sons Inc., Hoboken, NJ.

Wolfert, S., L. G. C. Verdouw, and M. Bogaardt. 2017. Big data in smart farming-A review. Agric. Syst. 153:69-80.

Zeiler, M. D. 2012. ADADELTA: An adaptive learning rate method. arXiv 1212.5701:1:6. http://arxiv.org/abs/1212.5701.

Zou, H., and T. Hastie. 2005. Regularization and variable selection via the elastic net. J. R. Stat. Soc. B 67:301-320. 